\section{L'axe PPAR $\gamma$-GDF3 dans les macrophages contrôle la fusion myogénique au cours de la régénération musculaire}

Rémi Mounier, Bénédicte Chazaud
Univ Lyon, Université Claude Bernard Lyon 1, CNRS UMR-5310, Inserm U-1217, Institut NeuroMyoGène, F-69622, Villeurbanne, France.

benedicte.chazaud@inserm.fr
Les macrophages sont des acteurs essentiels de la réparation tissulaire post-lésionnelle

La réponse inflammatoire à un dommage tissulaire - exempt d'infection bactérienne ou virale - est une réaction de l'organisme visant à limiter les dégâts lésionnels et permettre la réparation du tissu ou, quand cela n'est pas possible, sa cicatrisation. La réponse inflammatoire comporte une phase dite (pro) inflammatoire, suivie de la résolution de l'inflammation, qui permet l'engagement d'une phase anti-inflammatoire de réparation/cicatrisation. II a été montré dans de nombreux tissus que les macrophages jouent des rôles essentiels dans les deux phases de la réponse inflammatoire et qu'ils sont également importants dans l'étape clé de résolution de l'inflammation. Ceci est possible grâce à la grande versatilité phénotypique et fonctionnelle de ces cellules. En effet, dans tous les tissus étudiés jusqu'à présent, les macrophages inflammatoires dérivés des monocytes circulants qui infiltrent le tissu lésé, modifient leur profil inflammatoire au moment de la résolution de l'inflammation. Ils acquièrent alors un phénotype antiinflammatoire et participent activement à la phase de reconstruction du tissu.

Le muscle strié squelettique adulte est capable de se régénérer ad integrum. Après une lésion, les cellules souches, une fois activées, deviennent des progéniteurs capables d'expansion cellulaire puis de différenciation selon un programme de myogenèse permettant la reconstruction de nouvelles fibres musculaires

fonctionnelles. Les macrophages accompagnent ce processus très étroitement $[1](\rightarrow)$. Très rapidement après la lésion musculaire, les monocytes cir-

$(\rightarrow)$ Voir la Synthèse de S. Dufresne et al, $m / s n^{\circ} 6-7$, juin-juillet 2016, page 591 culants inflammatoires (de phénotype Ly6C ${ }^{p o s}$, positifs pour lymphocyte antigen 6 complex) infiltrent le muscle lésé et deviennent des macrophages inflammatoires qui stimulent la prolifération des progéniteurs myogéniques. À la suite de divers signaux, encore largement inconnus, mais au rang desquels la phagocytose des débris tissulaires semble jouer un rôle important, les macrophages inflammatoires réorientent leur statut inflammatoire pour acquérir un statut anti-inflammatoire ou de type réparateur (de phénotype Ly6C neg). Ces derniers stimulent les étapes suivantes de la myogenèse, à savoir l'engagement dans la myogenèse terminale et la fusion des cellules en structures plurinucléées qui préfigurent les nouvelles myofibres $[2,3]$. D'autres études ont montré que les macrophages contrôlent également la population de précurseurs fibrogéniques responsables du renouvellement de la matrice extracellulaire au cours de la phase de réparation du muscle strié squelettique [4].

Les voies métaboliques cellulaires contrôlent le statut inflammatoire des leucocytes

Le contrôle du changement de phénotype des macrophages et des fonctions des macrophages anti-inflammatoires réparateurs est très important afin que le tissu retrouve son intégrité. Des études de plus en plus nombreuses indiquent que les voies de régulation $\mathrm{du}$ métabolisme cellulaire participent à la régulation du statut inflammatoire des cellules de l'immunité [5], faisant émerger la notion «d'immunométabolisme ». Dans ce contexte, il a été montré qu'un des principaux senseurs énergétiques de la cellule, l'AMP kinase, qui s'active lorsque que le ratio AMP/ATP est trop élevé, est indispensable à la régénération musculaire en contrôlant le changement phénotypique des macrophages [6]. À l'inverse, le principal senseur de l'oxygène, le facteur induit par l'hypoxie HIF (hypoxia-inducible factor), n'est pas nécessaire dans les macrophages au cours de la régénération musculaire [7]. Plus globalement, le profil métabolique des populations macrophagiques Ly6C $C^{\text {pos }}$ et Ly6C ${ }^{\text {neg }}$ est altéré au cours de la régénération musculaire, avec, d'une façon générale, une augmentation des voies métaboliques mitochondriales [8]. Le métabolisme lipidique est également modifié dans les macrophages au cours de ce processus. Par ailleurs, il a été montré que le facteur de transcription régulé par les lipides, le PPAR $\gamma^{1}$ (peroxisome proliferator-activated receptor gamma), est fortement exprimé par les macrophages anti-inflammatoires Ly6Cneg au cours de la régénération musculaire post-lésionnelle [2]. PPAR $\gamma$ a été impliqué dans l'acquisition de certains

\footnotetext{
${ }^{1}$ PPAR $\gamma$ est un récepteur nucléaire qui agit comme facteur de transcription après liaison de ses ligands lipidiques, et qui joue un rôle majeur dans le métabolisme et l'adipogenèse.
} 


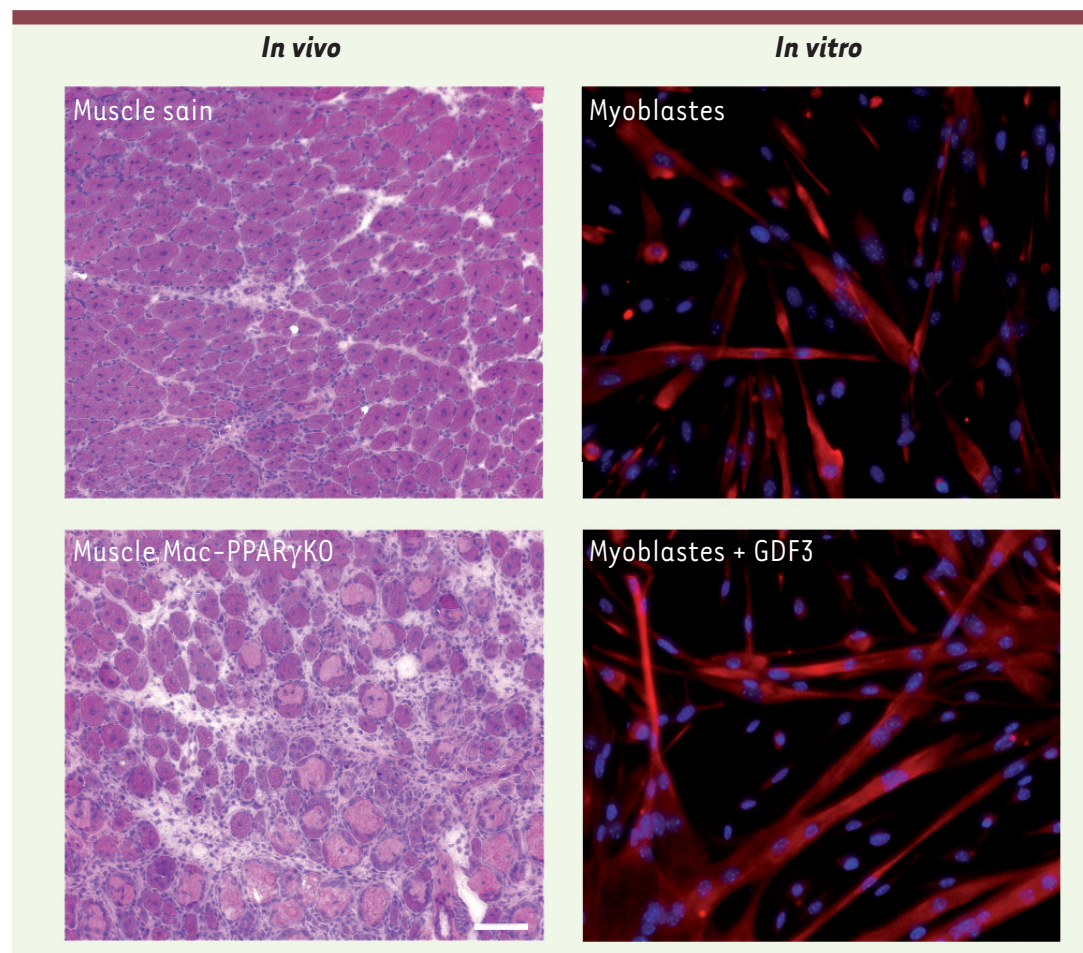

Figure 1. Rôle de l'axe PPAR $\gamma$-GDF3 dans la myogenèse adulte. À gauche, in vivo, des photos de section de muscle en régénération (coloration hémalun-éosine), 8 jours après une lésion induite par une toxine. En haut le muscle sain régénère normalement, avec la formation de nouvelles myofibres (structures violettes avec un ou plusieurs noyaux au centre). En bas le muscle déficient pour PPARy (peroxisome proliferator-activated receptor gamma) dans les macrophages montre un défaut majeur de la régénération avec la persistance de myofibres nécrotiques (larges structures rose pâle) et d'une inflammation importante (petites structures violet foncé). Barre $=100 \mu \mathrm{m}$. À droite, in vitro, en haut, des cellules myogéniques primaires de souris en culture forment des myotubes (structures multinucléées) qui préfigurent les nouvelles myofibres. En bas, les mêmes cellules traitées par du GDF3 (growth differentiation factor 3 ) recombinant montrent la formation de très larges myotubes contenant de nombreux noyaux, indiquant un effet stimulant du GDF3 sur la fusion des myoblastes. Les noyaux sont bleus et les cellules myogéniques sont marquées en rouge pour la desmine, un constituant du cytosquelette spécifique du muscle.

phénotypes anti-inflammatoires par les macrophages in vitro $[9,10]$, mais son rôle dans les phases de régénération/ cicatrisation tissulaire n'a jamais été documenté in vivo.

\section{PPAR $\gamma$ dans les macrophages est} requis pour la régénération musculaire Afin de déterminer le rôle du PPARy dans les macrophages, nous avons étudié la lignée de souris LysM-Cre;PPARyfl/fl (MacPPARYKO, pour knock-out pour PPAR $\gamma$ dans les macrophages), dans laquelle le gène PPAR $\gamma$ est spécifiquement invalidé dans la lignée myéloïde. La régénération musculaire post-lésionnelle est retardée et altérée durablement chez ces souris déficientes pour PPAR $\gamma$ dans les macrophages par rapport aux souris témoins. Des foyers inflammatoires sont observés 21 jours après la lésion et la taille des nouvelles fibres musculaires est sensiblement réduite, indiquant une mauvaise régénération musculaire (Figure 1). Des résultats similaires ont été obtenus avec des animaux chimères dont le système hématopoiétique a été reconstitué avec de la moelle osseuse provenant d'animaux déficients pour le gène PPAR $\gamma$.

L'analyse des sous-populations macrophagiques Ly $6 C^{\text {pos }}$ et Ly $6 C^{\text {neg }}$ chez les souris sauvages et Mac-PPAR $\gamma K O$ au cours de la régénération musculaire, indique que le facteur PPARy n'est pas requis pour le changement phénotypique des macrophages. Des expériences in vitro ont également montré que la déficience pour PPAR $\gamma$ n'altère pas les propriétés de phagocytose des macrophages. En revanche, les macrophages déficients pour PPAR $\gamma$ ont perdu certaines de leurs propriétés spécifiques vis-à-vis des progéniteurs myogéniques. En effet, alors que des macrophages normaux activés vers un statut anti-inflammatoire (traitement par les cytokines IL[interleukine]4 ou ILI0) stimulent la myogenèse et la fusion myogénique, les macrophages dépourvus de PPAR $\gamma$ ont complètement perdu ces propriétés et ne sont plus capables de stimuler la myogenèse.

\section{GDF3, cible de PPAR $\gamma$ dans les} macrophages anti-inflammatoires L'analyse comparée de la signature moléculaire des sous-populations de macrophages isolés du muscle de souris témoins ou invalidées pour PPARy (MacPPARYKO), à différents temps de régénération, a permis d'identifier le gène growth differentiation factor 3 ( $g d f 3$ ), comme étant fortement sous-exprimé dans les macrophages anti-inflammatoires (Ly6C ${ }^{\text {neg }}$ ) des souris Mac-PPARyKO. In vitro, la synthèse de GDF3 est activée par des ligands lipidiques synthétiques classiques de PPARy (comme la rosiglitazone) dans les macrophages Ly $6 \mathrm{C}^{\text {neg. In }}$ vivo, l'analyse de l'expression des lipides eicosanoïdes ${ }^{2}$ au cours de la régénération musculaire montre que la phase inflammatoire est caractérisée par la présence d'eicosanoïdes inflammatoires (PG[prostaglandine] E2, PGF2a), qui sont ensuite remplacés, pendant la phase réparatrice, par des médiateurs lipidiques comme 12-HETE (acide 12-hydroxyeicosatétraénoïque) et 15-HETE. Ces deux médiateurs lipidiques sont produits par la 12/15-lipoxygénase (Alox15), caractéristique de la phase anti-inflammatoire.

${ }^{2}$ Famille de lipides dérivés d'acides gras polyinsaturés à 20 atomes de carbone. 


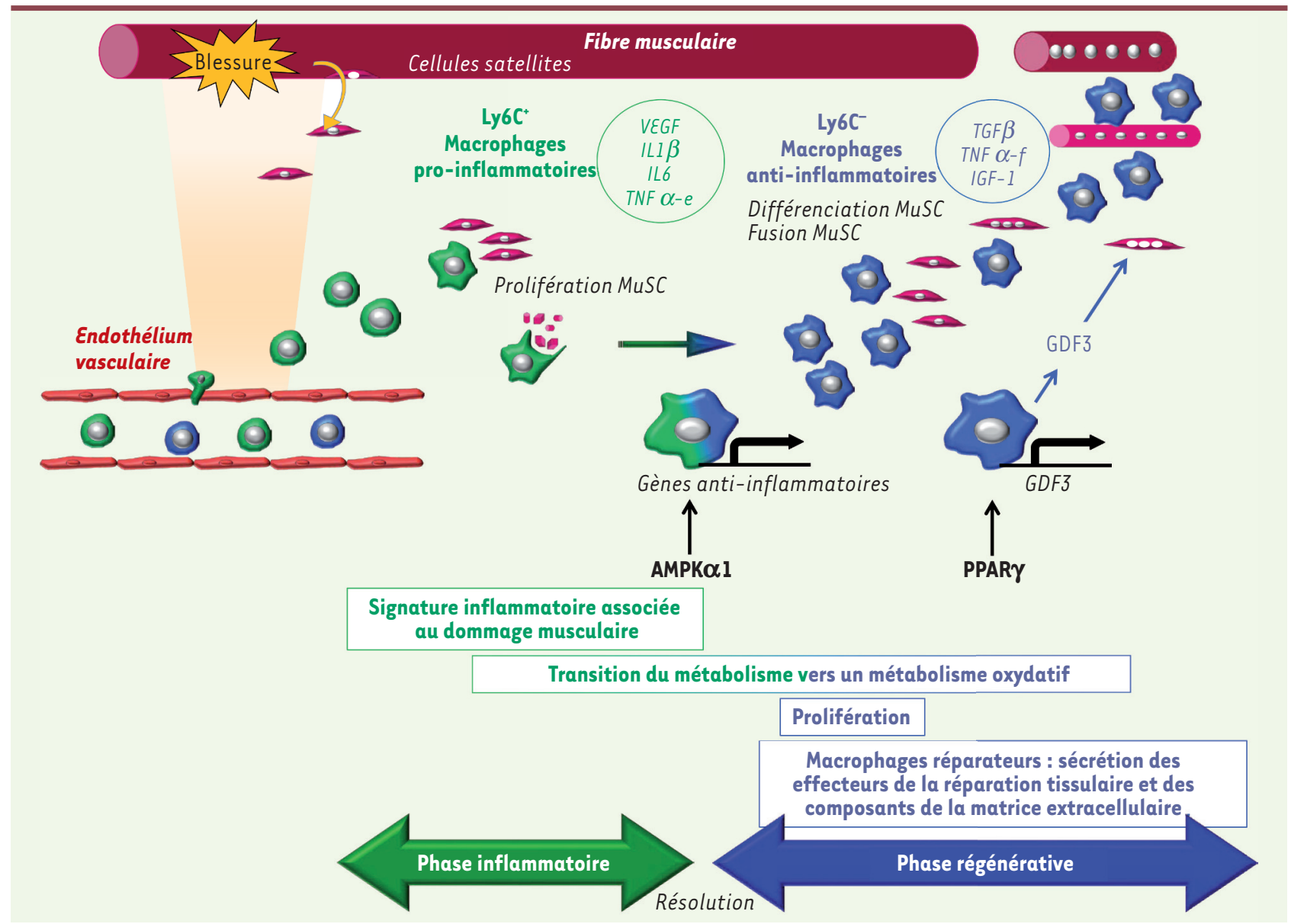

Figure 2. Schéma général de l'action des macrophages sur les cellules souches musculaires au cours de la régénération musculaire. Dès les premières étapes de la régénération, les monocytes Ly6C $C^{\text {pos }}$ (positifs pour lymphocyte antigen 6 complex) de la circulation entrent dans la zone lésée et se transforment en macrophages inflammatoires, caractérisés par une signature inflammatoire spécifique. Ces macrophages Ly6 $\mathrm{C}^{\mathrm{pos}}$ stimulent la prolifération et l'expansion des cellules souches musculaires (MuSC). La phagocytose des débris tissulaires et cellulaires, en association avec l'activation de la kinase AMPK $\alpha$ l (AMP kinase $\alpha$ l), permet d'enclencher la résolution de l'inflammation et l'acquisition par les macrophages d'un phénotype anti-inflammatoire Ly6 $\mathrm{C}^{\text {neg }}$ (négatifs pour lymphocyte antigen 6 complex). Ceci s'accompagne de changements métaboliques dans ces cellules. Les macrophages $\mathrm{Ly} 6 \mathrm{C}^{\text {neg }}$ anti-inflammatoires ou réparateurs prolifèrent et stimulent les dernières étapes de la myogenèse adulte, à savoir la différenciation myogénique et la fusion des cellules en myotubes/myofibres. Ceci est contrôlé par l'expression de PPAR (peroxisome proliferator-activated receptor gamma) dans les macrophages Ly6C $C^{\text {neg }}$ qui induit la sécrétion de GDF3 (growth differentiation factor 3). À son tour, GDF3 stimule spécifiquement la fusion des cellules myogéniques. Ainsi, la régénération musculaire suit une séquence coordonnée débutant par une phase inflammatoire suivie d'une phase de réparation. Au cours de ces phases, les macrophages exercent des rôles distincts grâce à l'acquisition de phénotypes inflammatoires spécifiques contrôlés par des régulateurs majeurs du métabolisme cellulaire. VEGF : vascular epidermal growth factor; ILI $\beta /$ IL6 : interleukine l $\beta / 6$; TNF $\alpha-e / f$ : tumor necrosis factor alpha, taux élevé/taux faible ; TGF $\beta$ : transforming growth factor- $\beta$; IGF- 1 : insulin growth factor.

Des analyses épigénomiques et génomiques, couplées à des expériences de CHIP-seq ${ }^{3}$ ont montré que le facteur PPAR $\gamma$ et le récepteur RXR (retinoid $X$ receptor) peuvent se lier sur 5 régions amplificatrices (enhancers)

${ }^{3}$ CHIP-seq est une méthode de séquençage (seq) de la chromatine (CH) immunoprécipitée (IP) par des anticorps spécifiques. Cette méthode permet de connaître les zones d'interaction des protéines sur l'ADN. au locus du gène $g d f 3$. La régulation de l'expression de gdf3 par PPAR $\gamma$ est confirmée par un niveau quasi-nul de l'expression de gdf3 dans les macrophages issus du muscle des souris MacPPARYKO. De plus, l'analyse comparée de divers types cellulaires isolés de muscle normal en régénération montre une expression exclusive de gdf3 par les macrophages.

\section{GDF3 sécrété par les macrophages} anti-inflammatoires stimule la fusion myogénique

In vivo, des souris chimères possédant un système hématopoïétique provenant de donneurs invalidés (KO) pour gdf3 montrent un défaut de régénération prononcé avec l'apparition de structures lipidiques en remplacement de certaines fibres et une taille réduite des nouvelles 
myofibres. L'injection intramusculaire du facteur de différenciation GDF3 recombinant restaure les défauts observés chez ces mêmes animaux. Ces résultats indiquent que le GDF3 secrété par les macrophages joue un rôle important dans la régénération musculaire. Afin d'en explorer les mécanismes, chaque étape de la myogenèse a été analysée en présence de GDF3 in vitro. L'ajout de GDF3 diminue légèrement la prolifération des précurseurs myogéniques et n'altère pas leur entrée dans le programme de différenciation terminale. En revanche, le facteur GDF3 stimule très fortement la dernière étape de la myogenèse, c'est-àdire la fusion des précurseurs différenciés en structures multinucléées ou myotubes, qui préfigurent les nouvelles myofibres (Figure 1).

L'ensemble de ces travaux [11] montre que PPAR $\gamma$ contrôle l'expression du facteur de différenciation GDF3 dans les macrophages dès le début de la phase anti-inflammatoire de la régénération musculaire (Figure 2). Le GDF3 secrété stimule spécifiquement la fusion des cellules myogéniques requise pour la formation de nouvelles myofibres. Ces données montrent un nouveau rôle d'un régulateur majeur du métabolisme cellulaire dans le contrôle des fonctions des macrophages anti-inflammatoires réparateurs, au cours de la régénération musculaire. $\diamond$

PPAR $\gamma$ transcription factor controls in anti-inflammatory macrophages the expression of GDF3 that stimulates myogenic cell fusion during skeletal muscle regeneration

\section{LIENS D'INTÉRÊT}

Les auteurs déclarent n'avoir aucun lien d'intérêt concernant les données publiées dans cet article.

\section{RÉFÉRENCES}

1. Dufresne SS, Frenette J, Dumont NA. Inflammation et régénération musculaire - Une arme à double tranchant. Med Sci (Paris) 2016 ; 32 : 591-7.

2. Arnold L, Henry A, Poron F, et al. Inflammatory monocytes recruited after skeletal muscle injury switch into antiinflammatory macrophages to support myogenesis. J Exp Med 2007 ; 204 : 1071-81.
3. Saclier M, Yacoub-Youssef H, Mackey AL, et al. Differentially activated macrophages orchestrate myogenic precursor cell fate during human skeletal muscle regeneration. Stem Cells $2013 ; 31: 384-96$.

4. Lemos DR, Babaeijandaghi F, Low M, et al. Nilotinib reduces muscle fibrosis in chronic muscle injury by promoting TNF-mediated apoptosis of fibro/ adipogenic progenitors. Nat Med 2015 ; 21 : 786-94

5. Norata GD, Caligiuri G, Chavakis T, et al. The cellular and molecular basis of translational immunometabolism. Immunity 2015 ; 43 : 421-34.

6. Mounier R, Theret M, Arnold L, et al. AMPKalphal regulates macrophage skewing at the time of resolution of inflammation during skeletal muscle regeneration. Cell Metab 2013; 18 : 251-64.

7. Gondin J, Theret M, Duhamel G, et al. Myeloid HIFs are dispensable for resolution of inflammation during skeletal muscle regeneration. J Immunol 2015 ; 194 : 3389-99.

8. Varga $T$, Mounier R, Horvath A, et al. Highly dynamic transcriptional signature of distinct macrophage subsets during sterile inflammation, resolution, and tissue repair. J Immunol 2016 ; 196 : 4771-82.

9. Huang JT, Welch JS, Ricote M, et al. Interleukin-4dependent production of PPAR-gamma ligands in macrophages by 12/15-lipoxygenase. Nature 1999 . $400: 378-82$

10. Bouhlel MA, Derudas B, Rigamonti $\varepsilon$, et al. PPARgamma activation primes human monocytes into alternative $\mathrm{m} 2$ macrophages with anti-inflammatory properties. Cell Metab 2007 ; 6 : 137-43.

11. Varga T, Mounier R, Patsalos A, et al. Macrophage PPARgamma, a lipid activated transcription factor controls the growth factor gdf3 and skeletal muscle regeneration the GDF3 and. Immunity 2016; 45 : 1038-51.

\section{NOUVELLE}

\section{Du neuf dans l'oncogenèse moléculaire des lymphomes: rôle du gène SETD2 dans les lymphomes T de l'intestin}

David Vallois ${ }^{1}$, Annalisa Roberti ${ }^{1}$, Bettina Bisig ${ }^{1}$, Philippe Gaulard ${ }^{2}$, Laurence de Leval ${ }^{1}$

\author{
${ }^{1}$ Institut de pathologie, \\ Centre hospitalier universitaire Vaudois, Lausanne, Suisse. \\ 2 Inserm U955, Université Paris-est, \\ département de pathologie, hôpital Henri-Mondor, \\ Créteil, France.
}

laurence.deLeval@chuv.ch

\section{Lymphomes T périphériques}

Les lymphomes T périphériques (LTP) dérivés de cellules T (ou NK, natural killer) matures regroupent une trentaine de maladies rares, qui représentent ensemble moins de $15 \%$ des hémopathies lymphoïdes malignes [1]. Ces néoplasies se manifestent sous forme de proliférations tissulaires, touchant les ganglions lymphatiques, la peau ou d'autres organes (région nasale, intestins, rate, etc.), ou de leucémies. La majorité des LTP non cutanés sont des maladies agressives, souvent résistantes aux polychimiothérapies classiques et ont un mauvais pronostic vital, avec une survie à 5 ans de moins de $30 \%$.

La pathogenèse des LTP est multifactorielle et complexe. Au plan génétique, jusqu'il y a peu, un très petit nombre de translocations ou de mutations récurrentes étaient connues. Récemment, l'analyse de ces tumeurs par différentes techniques de séquençage à haut débit a mis en évidence de nombreuses et diverses anomalies génétiques récurrentes, confirmant l'hétérogénéité moléculaire de ces maladies et mettant aussi en évidence l'implication de caractéristiques communes. Parmi les mutations les plus fréquentes, citons celles touchant différents modulateurs épigénétiques impliqués dans la méthylation de I'ADN, TET2 (Tet methylcytosine dioxygenase 2), DNMT3A (DNA [cytosine-5]-methyltransferase $3 A$ ) et IDH2 (isocitrate dehydro- 